\title{
Phase I/II Study: Experience with the Late Onset of Acute Pancreatitis after the Start of Chemotherapy with Gemcitabine Plus nab-Paclitaxel for Metastatic Pancreatic Cancer
}

\author{
Makoto Ueno ${ }^{1}$, Fumio Nagashima ${ }^{2}$, Hideki Ueno ${ }^{3}$, Masafumi Ikeda ${ }^{4}$, Shinichi Ohkawa ${ }^{1}$, \\ Nobumasa Mizuno ${ }^{5}$, Tatsuya Ioka ${ }^{6}$, Yasushi Omuro ${ }^{7}$, \\ Takako Eguchi Nakajima ${ }^{8}$ and Junji Furuse ${ }^{9}$
}

\begin{abstract}
:
Gemcitabine plus nab-paclitaxel is the current standard chemotherapy for patients with metastatic pancreatic cancer. We conducted a phase I/II study in Japan, in which high response rates and manageable toxicity were observed. In this study, two patients were reported as experiencing pancreatitis due to chemotherapy. In general, pancreatitis is sometimes observed when the tumor involves the pancreatic duct, and the onset is observed before the diagnosis or at the initial stage. The onset of pancreatitis in these cases was unique and observed after the start of chemotherapy. Pancreatitis may be induced by the alleviation of stenosis of the pancreatic duct associated with tumor shrinkage.
\end{abstract}

Key words: adverse event, gemcitabine, nab-paclitaxel, pancreatic adenocarcinoma, taxane

(Intern Med 58: 2957-2962, 2019)

(DOI: 10.2169/internalmedicine.2362-18)

\section{Introduction}

Metastatic pancreatic cancer is associated with a poor prognosis. The 5-year overall survival of patients with metastatic pancreatic cancer is $1-2 \%(1,2)$. Recently, based on results from phase III trials, the FOLFIRINOX and gemcitabine (GEM) plus nab-paclitaxel (nab-PTX) regimen has been approved and is used as a standard chemotherapy regimen for metastatic pancreatic cancer $(3,4)$. In Japan, a phase I/II study of GEM plus nab-PTX as chemotherapy for metastatic pancreatic cancer was performed from 2012 to 2015 to confirm the efficacy and safety. The results showed that GEM plus nab-PTX was associated with promising effi- cacy, and a response rate of $58.8 \%$ and median overall survival of 13.5 months, as well as acceptable toxicity, were observed (5).

Pancreatic cancer typically originates from the epithelium of the pancreatic duct and causes stenosis of the pancreatic duct and dilation of this duct in the caudal portion of the pancreas. In some patients who develop pancreatic cancer, pancreatitis is also observed before the diagnosis or at the initial stage; however, it typically recovers gradually with symptomatic treatment alone due to the atrophy of the caudal portion of the pancreas. We are unaware of any reports of the late onset of acute pancreatitis after chemotherapy for pancreatic cancer.

In the Japanese study, 2 of $34(5.9 \%)$ total enrolled pa-

${ }^{1}$ Division of Hepatobiliary and Pancreatic Oncology, Kanagawa Cancer Center, Japan, ${ }^{2}$ Department of Medical Oncology, Kyorin University Faculty of Medicine, Japan, ${ }^{3}$ Department of Hepatobiliary and Pancreatic Oncology, National Cancer Center Hospital, Japan, ${ }^{4}$ Department of Hepatobiliary and Pancreatic Oncology, National Cancer Center Hospital East, Japan, ${ }^{5}$ Department of Gastroenterology, Aichi Cancer Center Hospital, Japan, ${ }^{6}$ Department of Cancer Survey and Gastrointestinal Oncology, Osaka International Cancer Institute, Japan, ${ }^{7}$ Department of Medical Oncology, Tokyo Metropolitan Cancer and Infectious Diseases Center Komagome Hospital, Japan, ${ }^{8}$ Department of Medical Oncology, St. Marianna University School of Medicine Hospital, Japan and ${ }^{9}$ Department of Medical Oncology, Kyorin University Faculty of Medicine, Japan

Received: November 11, 2018; Accepted: February 27, 2019; Advance Publication by J-STAGE: June 27, 2019

Correspondence to Dr. Makoto Ueno, uenom@kcch.jp 


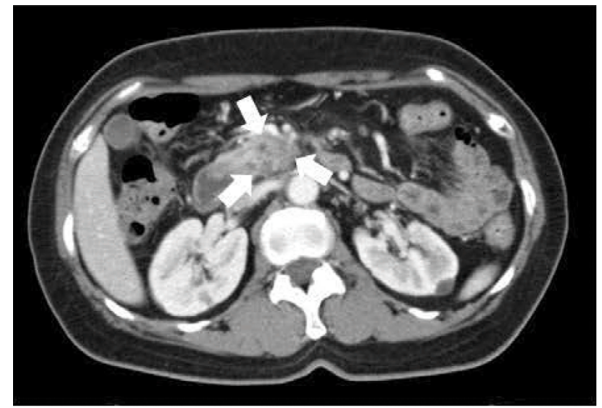

A

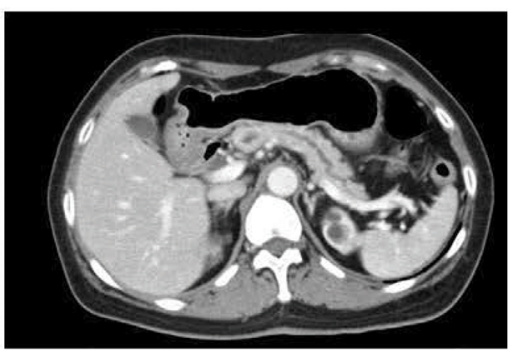

C

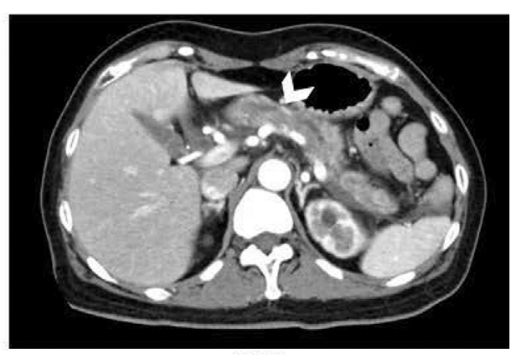

D2

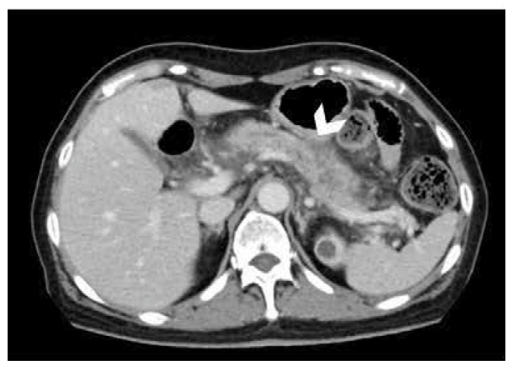

E

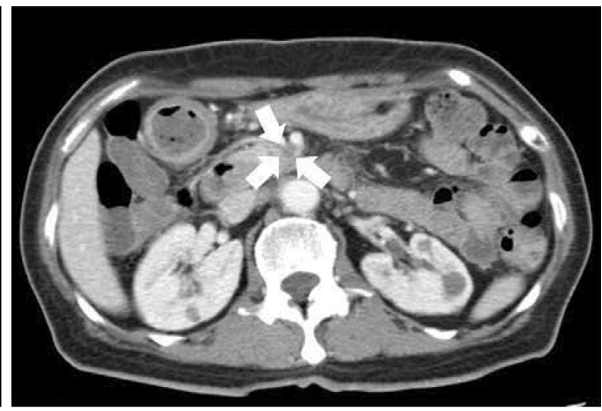

B

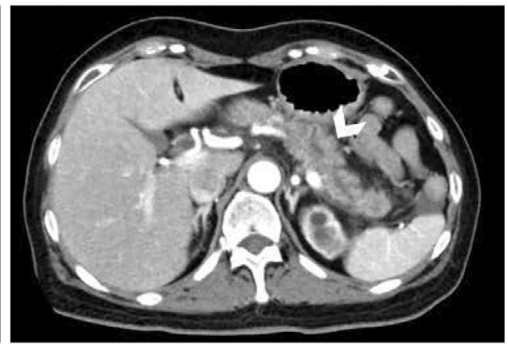

D1

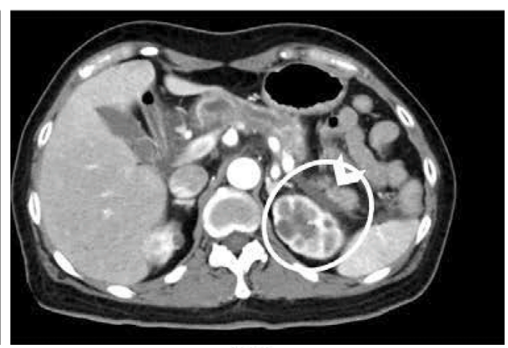

D3

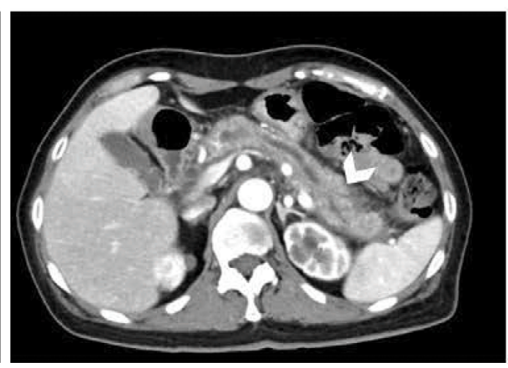

F

Figure 1. Computed tomography (CT) findings for Case 1. A: Pancreatic head cancer prior to chemotherapy, $22 \mathrm{~mm}$ in diameter (arrows). B: Pancreatic head cancer on Cycle 4 Day 21, $19 \mathrm{~mm}$ in diameter (best response) (arrows). C: The body and tail of the pancreas prior to chemotherapy. D1-3: The body and tail of the pancreas when Grade 3 pancreatitis (arrowheads) was observed on Cycle 2 Day 10. CT image of ascites (circle). E: The body and tail of the pancreas when Grade 3 pancreatitis (arrowhead) was observed on Cycle 2 Day 24. F: The body and tail of the pancreas when Grade 3 pancreatitis (arrowhead) was observed on Cycle 2 Day 32.

tients experienced the late onset of pancreatitis after the start of GEM plus nab-PTX. To our knowledge, this is the first report of the late onset of pancreatitis after the start of chemotherapy with GEM plus nab-PTX.

\section{Case Reports}

\section{Case 1}

A woman in her 60s was diagnosed with pancreatic head cancer with metastases to the lung and paraaortic lymph nodes. The pathological diagnosis of this patient was invasive ductal carcinoma. Reported complications included hy- 


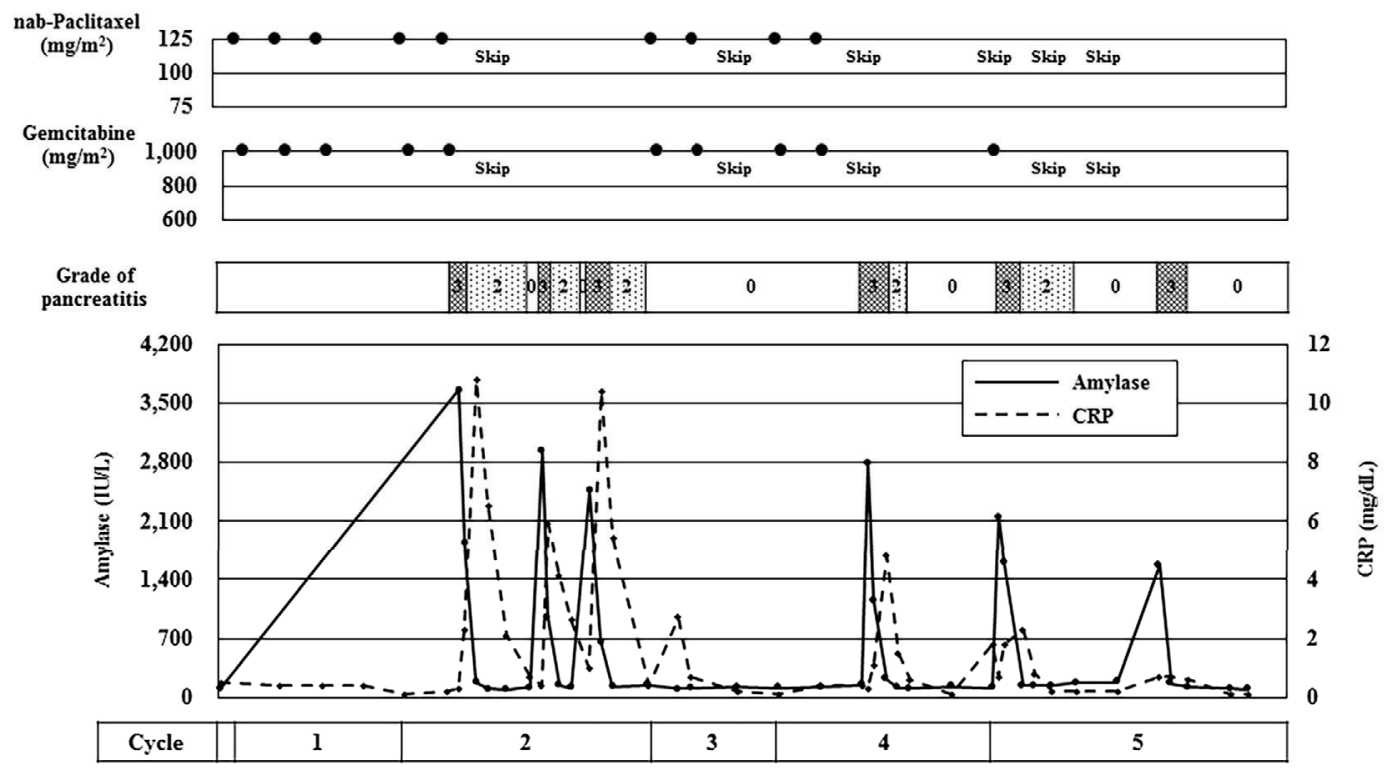

Figure 2. Changes in amylase values and the grade of pancreatitis for Case 1.

pertension (grade 3), constipation (grade 1) and hyperlipidemia (grade 1). Computed tomography (CT) with intravenous contrast revealed a pancreatic tumor measuring $22 \mathrm{~mm}$ in diameter, slight dilation of the pancreatic duct, and no atrophic pancreatic parenchyma (Fig. 1A and C). The patient received five cycles of GEM plus nab-PTX treatment. After five doses, the patient experienced pancreatitis (grade 3) as assessed by the Common Terminology Criteria for Adverse Events (CTCAE) version 4.0. In Cycle 2, the patient experienced grade 3 pancreatitis three times, as confirmed by CT (Fig. 1D-F).

The dosing regimen, changes in amylase values, Creactive protein (CRP) levels and the grade of pancreatitis are shown in Fig. 2. Serum amylase and CRP levels were elevated to $3,665 \mathrm{IU} / \mathrm{L}$ and $10.8 \mathrm{mg} / \mathrm{dL}$ respectively, at 1 month after the start of chemotherapy. CT showed a low density in the pancreas parenchyma with slight dilation of the pancreatic duct and acute necrotic collection (ANC). Nafamostat mesilate was administered to treat the pancreatitis, and the patient was instructed to temporarily fast; fluid replacement of $3 \mathrm{~L} /$ day was performed. After the initial onset of pancreatitis was resolved, GEM plus nab-PTX was resumed; however, acute pancreatitis with ANC continued to recur every few weeks, with nafamostat mesilate again administered in response. In Cycle 5, the patient was treated with GEM monotherapy. However, acute pancreatitis with ANC recurred again. Finally, the pancreatitis successfully resolved by one month after the onset of the last episode. Although the patient's response to the chemotherapy was stable disease based on the Response Evaluation Criteria in Solid Tumors (RECIST) v1.1, a $13.4 \%$ decrease in the sum of the longest diameters of the patient's primary lesions was observed (Fig. 1B).

\section{Case 2}

A man in his 50s was diagnosed with pancreatic head cancer with metastases to the liver. The patient had obstructive jaundice and had undergone placement of a covered metallic stent before the start of chemotherapy. The pathological diagnosis for the patient was invasive ductal carcinoma. Reported complications included cholestasis (grade 1). CT with intravenous contrast revealed a pancreatic tumor measuring $25 \mathrm{~mm}$ in diameter, slight dilation of the pancreatic duct and no atrophic pancreatic parenchyma (Fig. 3A and C).

The patient received 10 cycles of GEM plus nab-PTX treatment. Treatment was discontinued due to disease progression. After two months, the patient experienced a partial response according to RECIST v1.1 (Fig. 3B), and slight pancreatitis (grade 2) with ANC as assessed by CTCAE version 4.0 in the caudal portion of the pancreas was observed. Pancreatitis was also confirmed by CT (Fig. 3D1-4). Furthermore, CT showed a low-density area in the body of the pancreatic parenchyma as well as surrounding the tail of the pancreas. Gabexate mesilate and fluid replacement of $3 \mathrm{~L} /$ day were administered, and the patient was instructed to temporarily fast.

After resuming the administration of GEM plus nab-PTX, the pancreatitis (grade 3) recurred. Although pancrelipase and camostat mesilate were administered, the pancreatitis worsened (grade 4). It became associated with a walled-off necrosis (WON), and the CRP level increased to $29.9 \mathrm{mg} / \mathrm{dL}$ in Cycle 4 (Fig. 3E). Endoscopic ultrasound-guided cyst drainage was performed, and the patient's infection recovered. GEM plus nab-PTX was resumed and continued for 10 months with continuous cyst drainage. Although the pancreatitis recurred, severe pancreatitis did not develop (Fig. 3F). The dosing regimen, changes in amylase values 


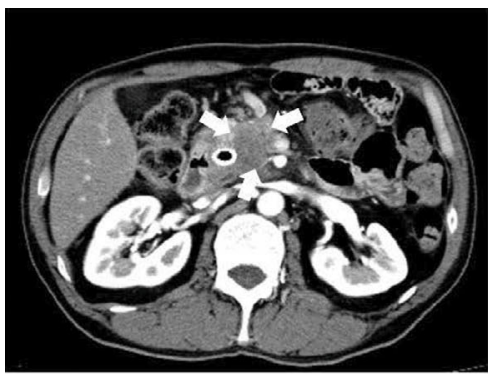

A

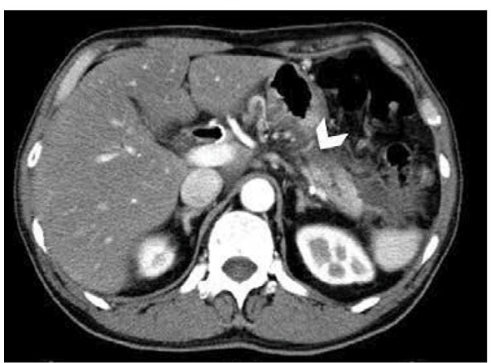

D1

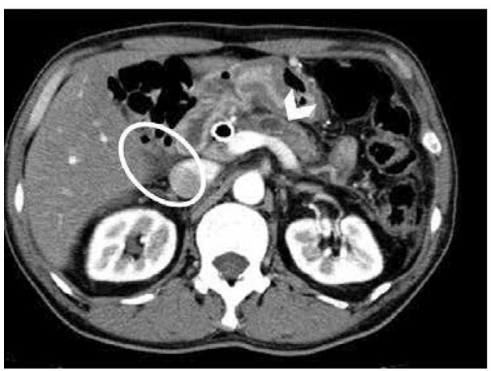

D3

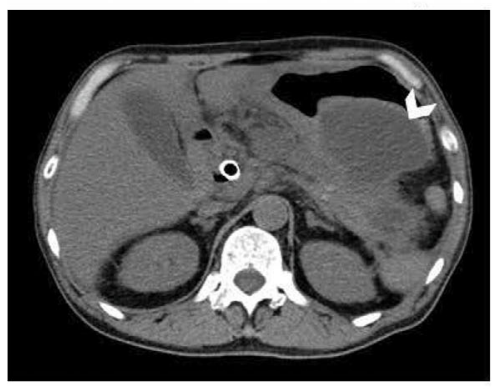

E

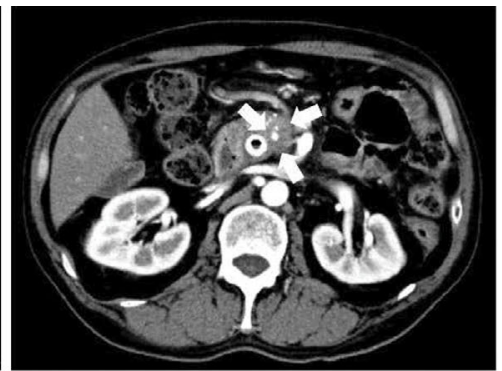

$\mathrm{B}$

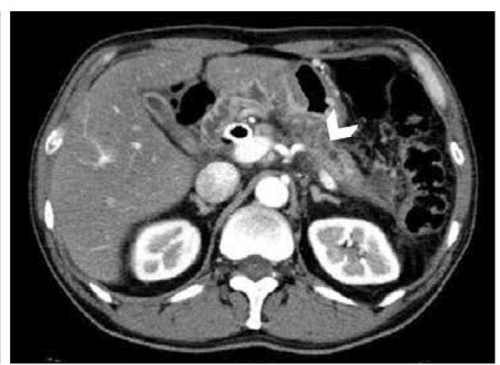

D2

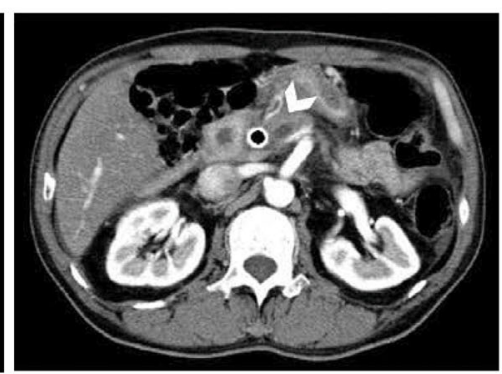

D4

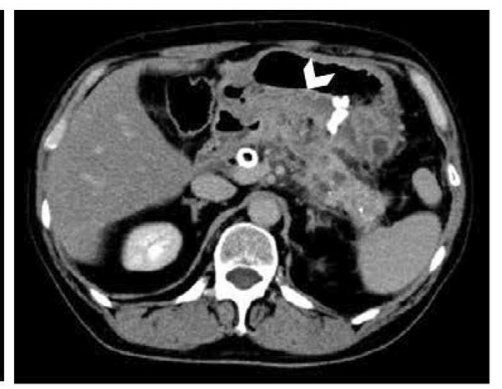

F

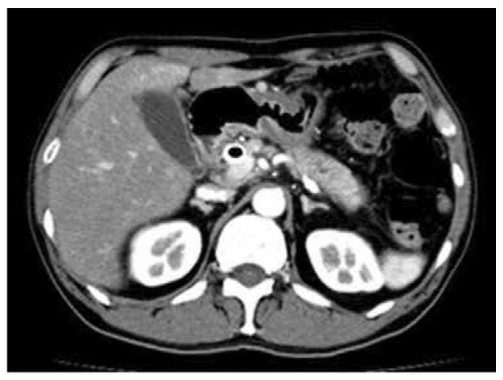

C

Figure 3. Computed tomography (CT) findings for Case 2. A: Pancreatic head cancer prior to chemotherapy, $25 \mathrm{~mm}$ in diameter (arrows). B: Pancreatic head cancer on Cycle 8 Day 16, 9 mm in diameter (best response) (arrows). C: The body and tail of the pancreas prior to chemotherapy. D1-4: The body and tail of the pancreas when Grade 2 pancreatitis (arrowheads) was observed on Cycle 3 Day 9. CT scan of ascites (circle). E: The body and tail of the pancreas when Grade 3 pancreatitis with WON (arrowhead) was observed on Cycle 4 Day 15. F: The body and tail of the pancreas when Grade 3 pancreatitis with continuous cyst drainage (arrowhead) was observed on Cycle 9 Day 23.

and CRP levels and the grade of pancreatitis are shown in Fig. 4.

\section{Discussion}

Pancreatitis caused by pancreatic cancer occurs on occasion, with a reported rate of $3 \%$ (6). In general, acute pancreatitis is sometimes induced when the pancreatic duct is stenosed due to pancreatic cancer. However, because of atrophy of the caudal pancreas, most patients do not develop pancreatitis, and pancreatitis usually occurs before the diagnosis or at the initial stage of pancreatic cancer. At later stages, the frequency of pancreatitis decreases due to the progression of pancreatic parenchyma atrophy. In the present two patients, pancreatitis was observed with a late onset, after several courses of chemotherapy. Such patients are very rare and are not typically observed after the start of chemotherapy for pancreatic cancer.

GEM plus nab-PTX was associated with a high response rate $(58.8 \%)$, and the waterfall plots showed significant tu- 


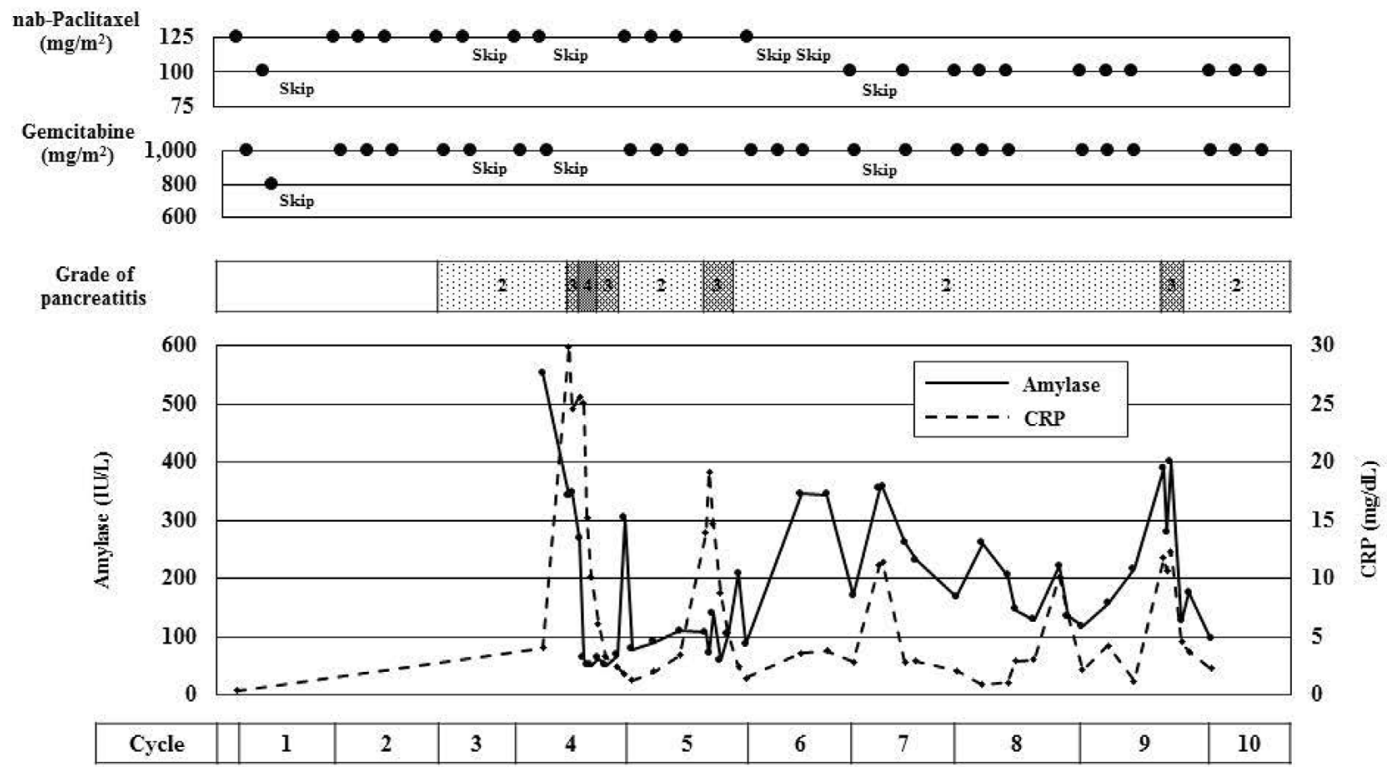

Figure 4. Changes in amylase values and the grade of pancreatitis for Case 2.

mor shrinkage. Tumor shrinkage rather than drug toxicity, patient predisposition or concomitant medications may have been responsible for the late onset of pancreatitis after starting chemotherapy with GEM plus nab-PTX. Acute pancreatitis may be induced by the alleviation of stenosis of the pancreatic duct associated with tumor shrinkage. In addition, restraint of the atrophy of the pancreatic parenchyma and pancreatic juice stagnation may have also been involved. In these two patients, slight dilation of the pancreatic duct was observed, and the pancreatitis seemed to recur easily. In addition, in Case 2, the presence of a biliary metallic stent seemed to worsen the pancreatitis (7).

Of note, there have been no reports of similar events of pancreatitis with FOLFIRINOX, which is an equally effective chemotherapy regimen. These pancreatitis cases were therefore thought to be associated with either pancreatic duct obstruction or drug-induced pancreatitis. The detailed reasons for the present findings are unclear due to the limited evidence available at present. There have been no reports of correlations between GEM plus nab-PTX and late onset of pancreatitis. However, there have been reports of improvement in pancreatic parenchyma fibrosis caused by GEM plus nab-PTX (8), which may improve the pancreatic function and be related to the late onset of pancreatitis.

\section{Conclusion}

Two patients experienced the late onset of pancreatitis after the start of GEM plus nab-PTX. As this condition can become serious, due consideration should be given to the risk of pancreatitis as a possible complication with GEM plus nab-PTX.

\section{Author's disclosure of potential Conflicts of Interest (COI).}

Makoto Ueno: Honoraria, Taiho Pharmaceutical. Fumio Nagashima: Honoraria, Taiho Pharmaceutical; Research funding, Taiho Pharmaceutical. Hideki Ueno: Honoraria, Taiho Pharma- ceutical. Masafumi Ikeda: Honoraria, Taiho Pharmaceutical. Shinichi Ohkawa: Honoraria, Taiho Pharmaceutical. Nobumasa Mizuno: Honoraria, Taiho Pharmaceutical; Research funding, Taiho Pharmaceutical. Tatsuya Ioka: Honoraria, Taiho Pharmaceutical; Research funding, Taiho Pharmaceutical. Takako Eguchi Nakajima: Honoraria, Taiho Pharmaceutical; Research funding, Taiho Pharmaceutical. Junji Furuse: Honoraria, Taiho Pharmaceutical; Research funding, Taiho Pharmaceutical.

\section{Financial Support}

This study (JapicCTI-121987) was sponsored by Taiho Pharmaceutical.

\section{Acknowledgement}

We would like to thank all of the patients and their families who participated in this study, as well as the investigators and their teams.

\section{References}

1. Sohal DP, Mangu PB, Khorana AA, et al. Metastatic pancreatic cancer: American Society of Clinical Oncology Clinical Practice Guideline. J Clin Oncol 34: 2784-2796, 2016.

2. Gong J, Tuli R, Shinde A, Hendifar AE. Meta-analyses of treatment standards for pancreatic cancer. Mol Clin Oncol 4: 315-325, 2016.

3. Conroy T, Desseigne F, Ychou M, et al. FOLFIRINOX versus gemcitabine for metastatic pancreatic cancer. N Engl J Med 364: 1817-1825, 2011.

4. Von Hoff DD, Ervin T, Arena FP, et al. Increased survival in pancreatic cancer with nab-paclitaxel plus gemcitabine. N Engl J Med 369: 1691-1703, 2013.

5. Ueno $\mathrm{H}$, Ikeda $\mathrm{M}$, Ueno $\mathrm{M}$, et al. Phase I/II study of nabpaclitaxel plus gemcitabine for chemotherapy-naive Japanese patients with metastatic pancreatic cancer. Cancer Chemother Pharmacol 77: 595-603, 2016.

6. Imamura M, Asahi S, Yamauchi H, Tadokoro K, Suzuki H. Minute pancreatic carcinoma with initial symptom of acute pancreatitis. J Hepatobiliary Pancreat Surg 9: 632-636, 2002. 
7. Isayama H, Nakai Y, Kogure H, Yamamoto N, Koike K. Biliary self-expandable metallic stent for unresectable malignant distal biliary obstruction: which is better: covered or uncovered? Dig Endosc 25 (Suppl): 71-74, 2013.

8. Von Hoff DD, Ramanathan RK, Borad MJ, et al. Gemcitabine plus nab-paclitaxel is an active regimen in patients with advanced pancreatic cancer: a phase I/II trial. J Clin Oncol 29: 4548-4554,
2011.

The Internal Medicine is an Open Access journal distributed under the Creative Commons Attribution-NonCommercial-NoDerivatives 4.0 International License. To view the details of this license, please visit (https://creativecommons.org/licenses/ by-nc-nd/4.0/).

(C) 2019 The Japanese Society of Internal Medicine Intern Med 58: 2957-2962, 2019 